\title{
Optimal Compensation of Harmonic Propagation in a Multi-Bus Microgrid
}

\author{
E. Skjong ${ }^{1,3,4}$ J. A. Suul ${ }^{2,5}$, M. Molinas ${ }^{1}$ and T.A. Johansen ${ }^{1,3}$ \\ ${ }^{1}$ Department of Engineering Cybernetics $\quad{ }^{2}$ Department of Electric Power Engineering \\ Norwegian University of Science and Technology \\ O. S. Bragstads plass, 7034 Trondheim, Norway \\ E-mail: espen.skjong@ulstein.com, jon.are.suul@ntnu.no, marta.molinas@ntnu.no, tor.arne.johansen@itk.ntnu.no \\ ${ }^{3}$ Centre for Autonomous Marine Operations and Systems, 7491 Trondheim, Norway \\ ${ }^{4}$ Ulstein Power \& Control AS, 6018 Ålesund, Norway \\ ${ }^{5}$ SINTEF Energy Research, 7465 Trondheim, Norway
}

\begin{abstract}
This paper discusses how an Active Power Filter (APF) can be utilized for system-wide harmonic mitigation in a microgrid with multiple sources of harmonic distortion located at different buses. A two-bus microgrid system with independent nonlinear loads at both buses is first investigated analytically, and it is demonstrated that it is possible to derive a harmonic current injection from the APF that will minimize the harmonic distortion at both buses. However, analytical optimization of the APF current will be sensitive to parameter variations, will deteriorate when the APF reaches current saturation and cannot be easily extended to larger systems with many loads at different buses. A more practically applicable method for calculating the APF current references, by using the framework of Model Predictive Control (MPC) is instead proposed for the investigated system. Under realistic operating conditions, this approach can obtain further improvement in the system-level harmonic mitigation. The characteristics and performances that are obtained with the analytical solution and the MPC-based control are assessed by time domain simulations in the Matlab/Simulink environment. The results clearly indicate how an MPC-based system-oriented compensation can maximize the utilization of a single APF in a multi-bus Microgrid.
\end{abstract}

\section{Keywords}

Active Power Filter, Harmonic Mitigation, Microgrid, Optimal Control, Power Quality

\section{Introduction}

Techniques for analyzing and mitigating harmonic distortions of voltage and current waveforms have been investigated since the early advents of electric power systems [1]. During the last decades, the impact of harmonic distortions has become more critical in industrial power systems due to the proliferation of Variable Speed Drives (VSDs) with diode- or thyristorbased rectifiers as grid interfaces. The dominant harmonics from various rectifier loads can be compensated by various passive filtering solutions, but such installations have limitations with respect to compensation of harmonics from dynamic loads and for operation in systems with time-varying resonance frequencies due to changes in the system configuration. However, the recent advances in switching converters have made Active Power Filters (APFs) a viable solution for real-time harmonic compensation under such conditions. As a result of extensive research activities, the converter design and the local control strategies of APFs for selective or broadband harmonic mitigation are also well established in literature [2], [3].

Today, APFs are commonly utilized for compensating the harmonic currents from a single load or for mitigating voltage distortions at a specific point in the power system. Thus, the real-time control methods for APFs are usually designed and developed on basis of only local considerations and local measurements. However, in systems with multiple sources of harmonic distortions, an APF has the potential to improve the system-level harmonic mitigation beyond what can be achieved based on only local considerations. The potential for systemoriented optimization of APF operation was realized in the early phase of research on power conditioning devices as analyzed in [4], but this aspect has not received significant attention in the development of APF control strategies. A first approach for system-level harmonic mitigation by using Model Predictive Control (MPC) for on-line optimization of the APF current references was recently proposed in [5]-[7].

Considering the potential for system-wide harmonic mitigation by a single APF, this paper analyzes a two-bus Microgrid system with independent nonlinear loads at both buses. For this system, it is shown that an explicit solution of the optimal APF compensation current for each harmonic component can be found analytically. The operation of the MPC-based approach from [5]-[7] is then compared to the performance that can be obtained with the analytical solution by time-domain simulations. The presented results demonstrate how the MPC-based operation achieves similar or better results than the analytical optimization, especially under the conditions when the APF reaches current saturation. The results are also benchmarked against traditional local control and the 


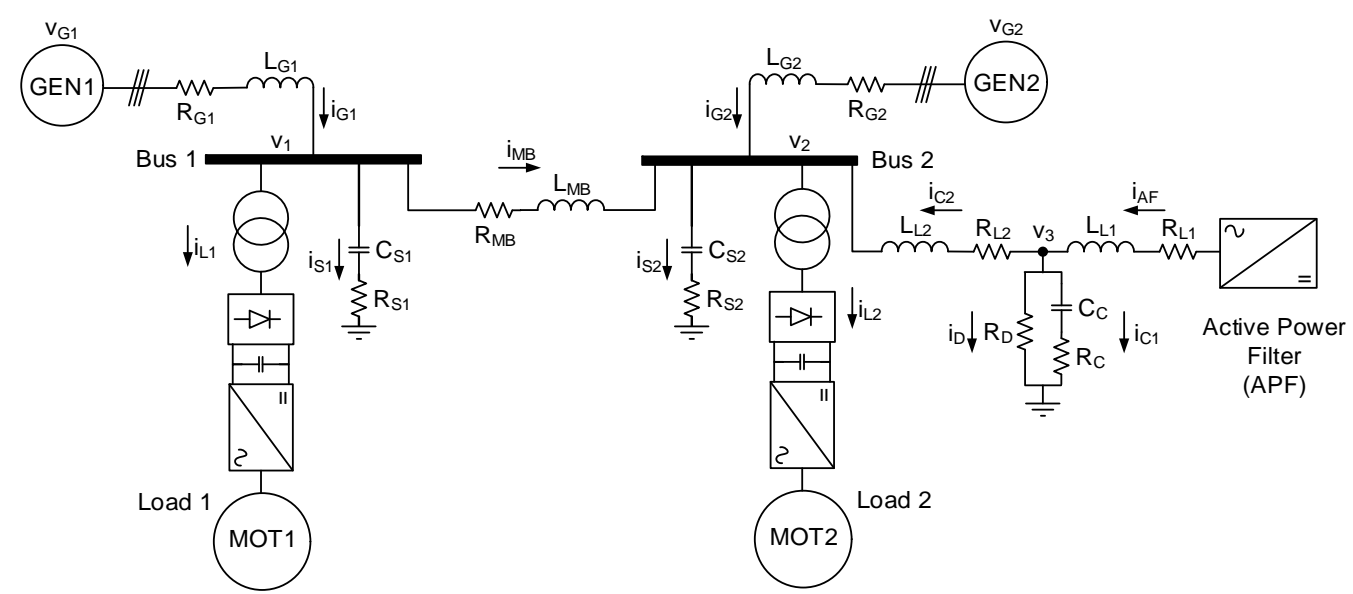

Fig. 1. Simplified shipboard power system (islanded microgrid) under investigation: Two generators, two buses with propulsion loads (Variable Speed Drive (VSD) with 12-pulse rectifiers), an Active Power Filter (APF) for harmonic mitigation, an LCL filter to suppress switching noise from the APF and RC-shunts to model parasitic shunt capacitance of cables and bus-bars.

control method from [8]. The results demonstrate how the system significantly benefits from control methods that are designed for system-wide harmonic mitigation.

\section{Investigated Microgrid Configuration}

The three-phase three-wire microgrid under investigation in this work is shown in Fig. 1. This system configuration can be considered to represent a two-split shipboard power system, with an Integrated Power System (IPS) configuration [9]. This system is in many aspects similar to other commercial islanded microgrids. The power system's two buses represent switchboards where one propulsion load and one generator are connected. The propulsion loads are realized as Variable Speed Drives (VSD) with 12-pulse rectifiers. The main bus connection between the two switchboards has an equivalent seriesimpedance given by $R_{M B}$ and $L_{M B}$. Parasitic shunt capacitances of the system are represented by $C_{S 1}$ and $C_{S 2}$ with equivalent resistances given by $R_{S 1}$ and $R_{S 2}$.

The 12-pulse rectifier loads are known to produce current harmonics with the $11^{\text {th }}, 13^{\text {th }}, 23^{\text {th }}, 25^{\text {th }}$ as the dominant components. Thus, in this work selective harmonic mitigation with the APF will be employed to mitigate the first four characteristic harmonic components generated by the 12-pulse rectifiers. The APF is interfaced to bus 2 with an LCL filter as indicated to the right in Fig. 1.

\section{Analysis of Optimal APF Currents}

Based on the power system showcased in Fig. 1, an analytical expression for the optimal APF current reference can be derived as a function of the harmonic load current.

\section{A. Derivation of Optimal APF Currents}

Using Kirchhoff's laws and assuming negligible harmonic distortion in the generator voltages, the quasi-stationary voltage and current components for each individual harmonic component in the system can be expressed by:

$$
i_{G 1,2}=-\frac{v_{1,2}}{R_{G 1,2}+j \cdot h \omega L_{G 1,2}}
$$

$$
\begin{aligned}
& i_{S 1,2}=-\frac{v_{1,2}}{R_{S 1,2}-\frac{1}{j \cdot h \omega C_{S 1,2}}} \\
& i_{M B}=-\frac{v_{1}-v_{2}}{R_{M B}+j \cdot h \omega L_{M B}} \\
& i_{C 2}=-\frac{v_{3}-v_{2}}{R_{L 2}+j \cdot h \omega L_{L 2}} \\
& v_{3}=\left(R_{C}-\frac{1}{j \cdot h \omega C_{C}}\right) i_{C 1}=R_{D} i_{D} \\
& i_{A F}=i_{C 1}+i_{C 2}+i_{D} \\
& i_{G 2}=i_{L 2}+i_{S 2}-i_{C 2}-i_{M B} \\
& i_{G 1}=i_{S 1}+i_{L 1}+i_{M B}
\end{aligned}
$$

The current and voltage components in these equations are defined in Fig. 1. The equations are expressed on phasor form where $j$ is the imaginary operator and $h$ is the harmonic order. The system is expressed by 11 equations (with 11 unknowns) for each harmonic component. This set of equations can be solved analytically for the harmonic currents flowing in the generators as a function of the harmonic load currents and the current from the APF. Symbolic mathematical software, such as Maple ${ }^{1}$, can be used to find a solution for the system given $\mathrm{as}^{2}$ :

$$
\chi=\gamma\left(\rho, \omega, h, i_{L 1}, i_{L 2}, i_{A F}\right)
$$

The 11 unknown variables are:

$$
\boldsymbol{\chi}=\left[i_{G 1}, i_{G 2}, i_{S 1}, i_{S 2}, i_{M B}, i_{C 1}, i_{C 2}, i_{D}, v_{1}, v_{2}, v_{3}\right]^{\cdot}
$$

while the power system parameters collected in the vector $\boldsymbol{\rho}$ are given by (11).

$$
\begin{array}{r}
\boldsymbol{\rho}=\left[L_{G 1}, R_{G 1}, L_{G 2}, R_{G 2}, L_{M B}, R_{M B}, C_{S 1}, R_{S 1}, \ldots\right. \\
\left.C_{S 2}, R_{S 2}, L_{L 1}, R_{L 1}, L_{L 2}, R_{L 2}, C_{C}, R_{C}, R_{D}\right]
\end{array}
$$

The purpose of the harmonic mitigation should be to minimize the harmonic components in bus voltages and the generator currents. In this case, an objective function for minimizing the currents in the generators for a general harmonic order $h$ is selected as given by (12):

\footnotetext{
${ }^{1} \mathrm{http}: / /$ www.maplesoft.com

2 The detailed solution is too large to show in this paper.
} 

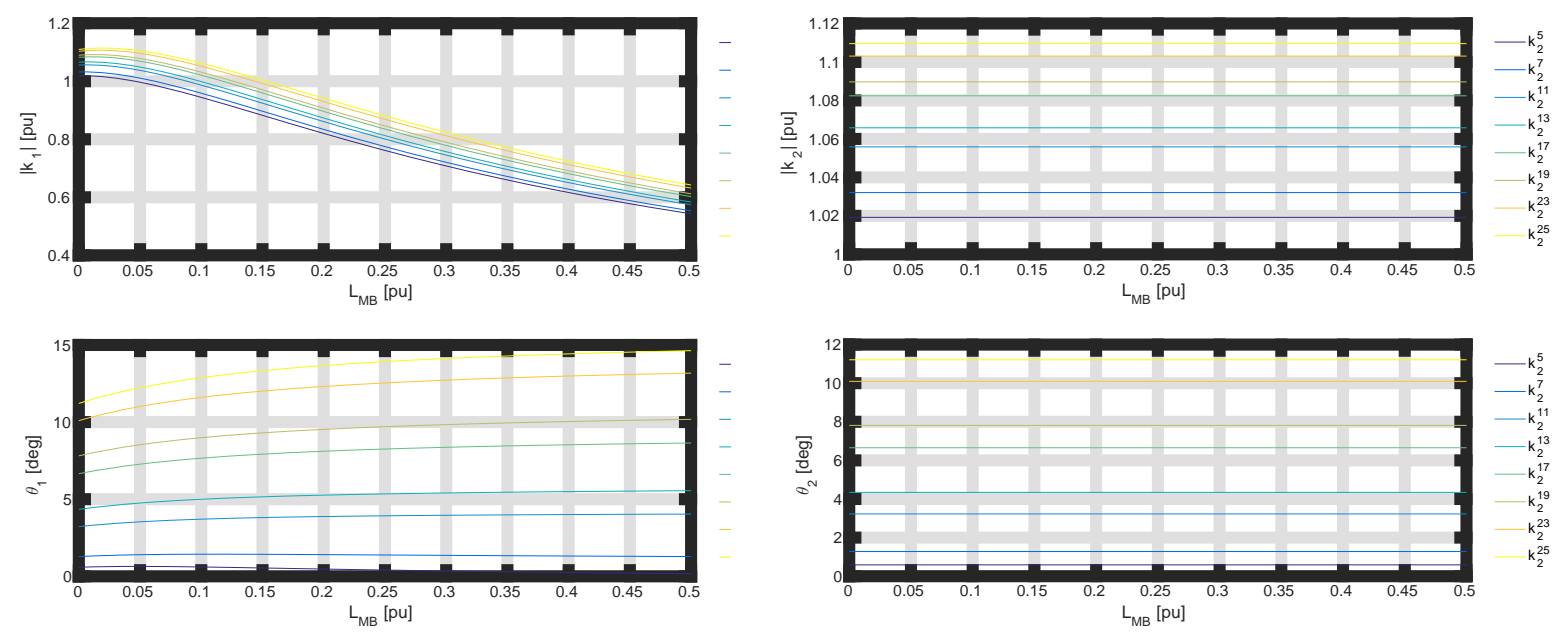

Fig. 2. Magnitude (a) and angle (b) of $k_{1}$ and $k_{2}$ plotted for eight first harmonics $\left(5^{\text {th }}, 7^{\text {th }}, 11^{\text {th }}, 13^{\text {th }}, 17^{\text {th }}, 19^{\text {th }}, 23^{\text {rd }}, 25^{\text {th }}\right)$ as a function of the

Fig. 2. Magnitude (a) and angle (b) of $k_{1}$ and $k_{2}$ plotted for eight first harmonics $\left(5^{\text {th }}, 7^{\text {th }}, 11^{\text {th }}, 13^{\text {th }}, 17^{\text {th }}, 19^{\text {th }}, 23^{\text {rd }}, 25^{\text {th }}\right)$ as a function of the main bus inductance $L_{M B}$

$$
\min _{i_{A F}} J\left(\rho, \omega, h, i_{L 1}, i_{L 2}, i_{A F}\right)=\left|i_{G 1}\right|^{2}+\left|i_{G 2}\right|^{2}
$$

The analysis is assuming that the optimal APF current is given on the form:

$$
i_{A F}^{c}=i_{a f}^{r e}+j \cdot i_{a f}^{i m}
$$

considering both magnitude and phase information. Substituting (13) for $i_{A F}$ in the solution of $i_{G 1}$ and $i_{G 2}$ resulting from (12), the minimization problem can be solved by differentiation with respect to the real and imaginary part of $i_{A F}^{c}$ :

$$
\begin{aligned}
& \frac{d J}{d i_{a f}^{r e}}\left(\boldsymbol{\rho}, \omega, h, i_{L 1}, i_{L 2}, i_{A F}^{c}\right)=0 \\
& \frac{d J}{d i_{a f}^{i m}}\left(\boldsymbol{\rho}, \omega, h, i_{L 1}, i_{L 2}, i_{A F}^{c}\right)=0
\end{aligned}
$$

This yields a solution on the form

$$
\begin{aligned}
i_{A F}^{o}=k_{1} \cdot i_{L 1}^{h} & +k_{2} \cdot i_{L 2}^{h} \\
= & \left|k_{1}(\rho, \omega, h)\right| i_{L 1}^{h} \cdot e^{-j \cdot \theta_{1}(\rho, \omega, h)} \\
& +\left|k_{2}(\rho, \omega, h)\right| \cdot i_{L 2}^{h} \cdot e^{-j \cdot \theta_{2}(\rho, \omega, h)}
\end{aligned}
$$

where $\theta_{1}=\angle k_{1}, \theta_{2}=\angle k_{2}$, and $i_{L 1}^{h}, i_{L 2}^{h}$ are the load currents for harmonic $h$. Thus, the constants $k_{1}$ and $k_{2}$ are defining the optimal APF current given as a function of $i_{L 1}^{h}, i_{L 2}^{h}$ and the system parameters $\boldsymbol{\rho}$.

\section{B. Analysis of Optimal AFP Currents}

To illustrate the results from the analytical optimization, the system configuration from Fig. 1 is analyzed with the parameters in Table I. Fig. 2 shows the magnitude and angles of $k_{1}$ and $k_{2}$ plotted as functions of $L_{M B}$. As can be seen, both the angle and the magnitude of $k_{l}$ are dependent on $L_{M B}$ while the angle and the magnitude of $k_{2}$ are independent on $L_{M B}$. This is as expected since current injection at bus 2 for mitigating harmonics in $i_{G I}$ must be corrected for the voltage drop and phase shifts introduced by the main bus impedance and the shunt capacitances of the system. The presented curves also indicate how the optimization balances the distortion in the two buses depending on the impedance between them. In case the bus impedance is small, the APF can compensate for the harmonics of both loads, while the compensation of the harmonics from load 1 has to be reduced when the bus
Table I: Power System Parameters (pu rel. generator rating)

\begin{tabular}{lll}
\hline Parameter & Value & Unit \\
\hline Nominal voltage $V_{r m s}$ & 690 & $\mathrm{~V}$ \\
Nominal frequency $f$ & 50 & $\mathrm{~Hz}$ \\
Generator power rating & 1 & $\mathrm{MVA}$ \\
\hline$L_{G 1}$ and $L_{G 2}$ & 0.2 & {$[\mathrm{pu}]$} \\
$R_{G 1}$ & $0.1 \cdot L_{G 1} \cdot \omega$ & {$[\mathrm{pu}]$} \\
$R_{G 2}$ & $0.1 \cdot L_{G 2} \cdot \omega$ & {$[\mathrm{pu}]$} \\
$L_{M B}$ & 0.04 & {$[\mathrm{pu}]$} \\
$R_{M B}$ & $0.1 \cdot L_{M B} \cdot \omega$ & {$[\mathrm{pu}]$} \\
\hline$C_{S 1}$ and $C_{S 2}$ & 2 & $\mu \mathrm{F}$ \\
$R_{S 1}$ and $R_{S 2}$ & 2 & $\Omega$ \\
$L_{L 1}$ and $L_{L 2}$ & 0.3 & $\mathrm{mH}$ \\
$R_{L 1}$ and $R_{L 2}$ & 0.03 & $\Omega$ \\
$C_{C}$ & 30 & $\mu \mathrm{F}$ \\
$R_{C}$ & 10 & $\Omega$ \\
$R_{D}$ & 160 & $\Omega$ \\
\hline
\end{tabular}

impedance increases to avoid increased distortion in $i_{G 2}$. However, the result from this optimization is only valid as long as the system parameters are accurately known and the current rating of the APF is not exceeded.

\section{MPC Generated APF Reference}

Another option for generating an optimal APF current reference in a multi-bus system configuration was proposed in [5]-[7], utilizing an online optimization scheme based on Model Predictive Control (MPC). Fig. 3 illustrates the main concept of MPC, where measurements from the process are initializing a model of the system which is used to obtain the control action at each sampling interval by solving a finite horizon optimal control problem [10]. The objective of the optimization is formulated as a cost function which is given to an optimizer together with constraints reflecting limitations of the physical process. The control actions are optimized according to a predefined reference $r\left(t_{k}\right)$ (with discretized time step $t_{k}$ ) reflecting the desired process behavior. The model output is given by $\hat{y}\left(t_{k}\right)$, and a closed-loop feedback provides the necessary model corrections $\varepsilon\left(t_{k}\right)$ calculated as model mismatch between the outputs of the model, $\hat{y}\left(t_{k}\right)$ and the process, $y\left(t_{k}\right)$.

The total MPC formulation can be expressed on a standardized form given by (16). In this equation the dynamic state vector of the process is given by $\mathbf{x}(t)$, the 


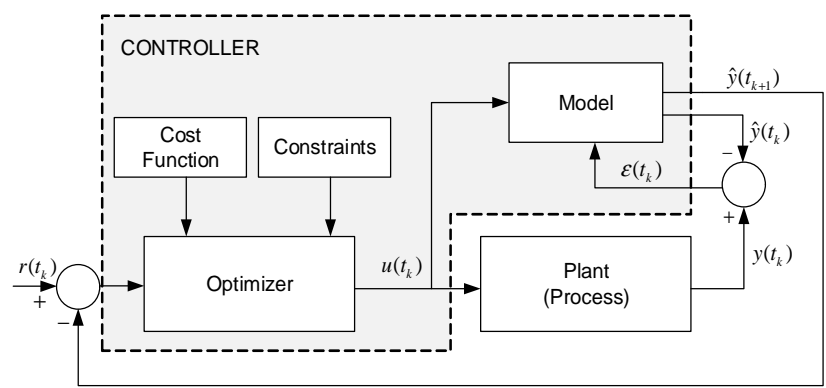

Fig. 3. General illustration of a Model Predictive Controller.

algebraic state vector is $\mathbf{z}(t)$, and the optimal control vector is $\mathbf{u}(t)$. The scalar function $V(\cdot)$ is the objective function (with $l(\cdot)$ as stage cost). The differential states are given by $\mathbf{f}(\cdot)$, the algebraic states and equality constraints are given by $\mathbf{g}(\cdot)$. Inequality constraints are given by $\mathbf{h}(\cdot)$. The length of the discrete control horizon is defined by $T$, where $t_{0}$ is the initial time instance, and the optimal solution's feasible region is given by $\mathrm{S}$. The power system's measurements $\left(y\left(t_{k}\right)\right.$ in Fig. 3) are used to initialize the MPC, i.e. $\mathbf{x}\left(t_{0}\right)$ and $\mathbf{z}\left(t_{0}\right)$.

$\min _{\mathbf{x}(t), \mathbf{z}(t), \mathbf{u}(t)} V(\mathbf{x}(t), \mathbf{z}(t), \mathbf{u}(t))=\int_{t_{0}}^{t_{0}+T} l(\mathbf{x}(t), \mathbf{z}(t), \mathbf{u}(t)) d t$

s.t.

$\dot{\mathbf{x}}(t)=\mathbf{f}(\mathbf{x}(t), \mathbf{z}(t), \mathbf{u}(t))$

$\mathbf{g}(\mathbf{x}(t), \mathbf{z}(t), \mathbf{u}(t))=0$

$\mathbf{h}(\mathbf{x}(t), \mathbf{z}(t), \mathbf{u}(t)) \leq 0$

$\forall t \in\left[t_{0}, t_{0}+T\right] \wedge \mathbf{x}\left(t_{0}\right), \mathbf{z}\left(t_{0}\right) \in \mathrm{S}$,

The simplified power system model used in the derivation of the MPC is illustrated in Fig. 4. Compared to the system model in Fig. 1, the LCL filter has been ignored and the RC-shunt elements are replaced by simple Cshunts. The applied MPC formulation, based on [5]-[7], is briefly addressed in the following.

\section{A. Model Formulation}

Using Kirchhoff's laws, the dynamics of the power system represented in Fig. 4 can be expressed as

$$
\begin{gathered}
L_{G 1,2} \frac{d \mathbf{i}_{G 1,2}}{d t}=-R_{G 1,2} \mathbf{i}_{G 1,2}-\mathbf{v}_{S 1,2} \\
C_{S 1} \frac{d \mathbf{v}_{S 1}}{d t}=\mathbf{i}_{G 1}-\mathbf{i}_{M B}-\mathbf{i}_{L 1} \\
C_{S 2} \frac{d \mathbf{v}_{S 2}}{d t}=\mathbf{i}_{M B}+\mathbf{i}_{G 2}-\mathbf{i}_{L 2}+\mathbf{i}_{A F} \\
L_{M B} \frac{d \mathbf{i}_{M B}}{d t}=\mathbf{v}_{S 1}-\mathbf{v}_{S 2}-R_{M B} \mathbf{i}_{M B}
\end{gathered}
$$

In these equations, three-phase currents and voltages are given by bold symbols, i.e. i and $\mathbf{v}$. As the MPC formulation is only considering the harmonic currents and voltages, the generator voltages are not included in the model since they are assumed to have no harmonic voltage components. The load currents can be written as Fourier series according to (21), with amplitudes $I_{L, i}$, phases $\phi_{L, i}$ and harmonic orders to be mitigated given by $i$. Note that the fundamental frequency load current components are not included since only the harmonic currents are object of mitigation.

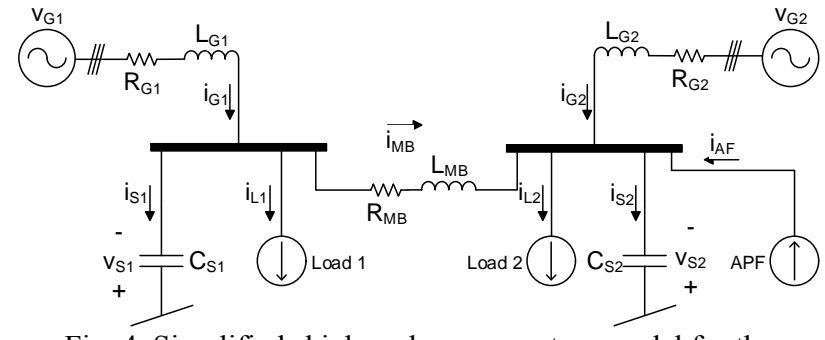

Fig. 4. Simplified shipboard power system model for the derivation of the MPC.

$$
\mathbf{i}_{L}(t)=\left[\begin{array}{c}
i_{L, a}(t) \\
i_{L, b}(t) \\
i_{L, c}(t)
\end{array}\right]=\left[\begin{array}{c}
\sum_{i} I_{L, i} \sin \left(i\left(\omega t+\phi_{L, i}\right)\right) \\
\sum_{i} I_{L, i} \sin \left(i\left(\omega t+\phi_{L, i}-\frac{2 \pi}{3}\right)\right) \\
\sum_{i} I_{L, i} \sin \left(i\left(\omega t+\phi_{L, i}+\frac{2 \pi}{3}\right)\right)
\end{array}\right],
$$

$\forall i \in\{$ harmonic orders $\}$

The APF reference currents, $\mathbf{i}_{A F}$, are kept as free variables and decided by the optimization scheme. With regards to MPC standard form, the dynamic and algebraic state vectors ( $(\mathbf{x}$ and $\mathbf{z})$, along with the control vector (u) can be written as

$$
\begin{aligned}
& \mathbf{x}=\left[\ddot{\mathbf{i}}_{G 1}, \mathbf{i}_{G 2}, \mathbf{i}_{M B}, \mathbf{v}_{S 1}, \mathbf{v}_{S 2}\right] \\
& \mathbf{z}=\left[\mathbf{v}_{G 1}, \mathbf{v}_{G 2}, \ddot{i}_{L 1}, \dot{i}_{L 2}\right]^{\cdot} \\
& \mathbf{u}=\mathbf{i}_{A F}=\left[i_{A F, a}, i_{A F, b}, i_{A F, c}\right]
\end{aligned}
$$

\section{B. Cost Function}

A suitable stage cost function for harmonic mitigation in the two switchboards can be stated as:

$$
\begin{aligned}
& l(\mathbf{x}, \mathbf{z}, \mathbf{u})=\ddot{\mathbf{i}}_{G 1} \mathbf{Q}_{\mathbf{1}_{G 1}}+\ddot{\mathbf{i}}_{G 2} \mathbf{Q}_{2} \mathbf{i}_{G 2}+\mathbf{u}^{\cdot} \mathbf{Q}_{u} \mathbf{u} \\
& +\left(\mathbf{i}_{A F, a}+\mathbf{i}_{A F, b}+\mathbf{i}_{A F, c}\right)^{\circ} \mathbf{Q}_{a b c}\left(\mathbf{i}_{A F, a}+\mathbf{i}_{A F, b}+\mathbf{i}_{A F, c}\right),
\end{aligned}
$$

with weights given by

$$
\begin{aligned}
& \mathbf{Q}_{1}=\operatorname{diag}\left(\left[q_{1}, q_{1}, q_{1}\right]\right), \mathbf{Q}_{2}=\operatorname{diag}\left(\left[q_{2}, q_{2}, q_{2}\right]\right), \\
& \mathbf{Q}_{u}=\operatorname{diag}\left(\left[q_{u}, q_{u}, q_{u}\right]\right), \mathbf{Q}_{a b c}=\operatorname{diag}\left(\left[q_{a b c}, q_{a b c}, q_{a b c}\right]\right) .
\end{aligned}
$$

The first two parts of the stage cost function given by (23) is related to harmonic mitigation and penalizes harmonic currents not equal to zero. The two last parts penalizes for high APF current amplitude and zero sequence components.

\section{Constraints}

The MPC's constraints should reflect the properties and limitations of the physical process, and in this work the constraints are related to the APF current limitations. The blue hexagon in Fig. 5 illustrates the phase current limitations of the APF [11]. Assuming a balanced filter, the limits will be the same for all phases as expressed by.

$$
\begin{aligned}
& -i_{A F}^{a p} \leq i_{A F, a} \leq i_{A F}^{a p} \\
& -i_{A F}^{a p} \leq i_{A F, b} \leq i_{A F}^{a p} \\
& -i_{A F}^{a p} \leq i_{A F, c} \leq i_{A F}^{a p}
\end{aligned}
$$

The constraints are added to the function $\mathbf{h}(\cdot)$ in the MPC formulation on standard form. 


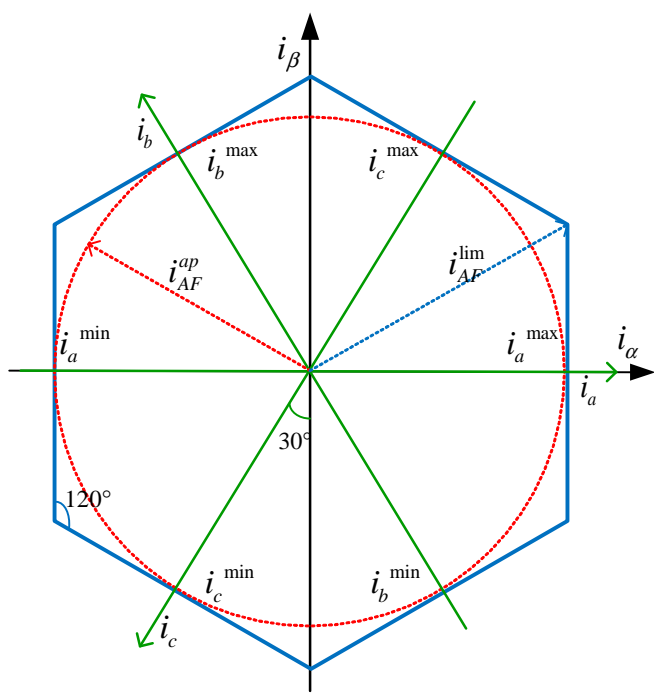

Fig. 5. Active power filter current limitation for three-phase three-wire system represented in the abc and $\alpha \beta$ frames [11]

\section{Simulation study}

The power system from Fig. 1 has been modelled in the Matlab/Simulink environment using the SimPowerSystems library. For simplicity, the generators are assumed to be ideal voltage sources and the frequency is assumed to be constant. The APF is operated with inner loop hysteresis current controllers with $15 \%$ hysteresis band and switching frequency of approximately $20 \mathrm{kHz}$. The APF's power rating is set to $15 \%$ of the rating of the generators. The simulation step size is set to $2 \mu$ s. The MPC is implemented using the ACADO (Automatic Control and Dynamic Optimization) toolkit [12], which comes with a code generation tool [13] for generating highly effective $\mathrm{C}$ code. The MPC was cross-compiled to Matlab (as a mex-function), and included in the Simulink implementation of the power grid. Details regarding the MPC implementation are listed in Table II.

Two different cases are simulated to illustrate the performance of the MPC compared to results when operating the APF with current reference according to (15) with constants $k_{1}$ and $k_{2}$ obtained from analytical optimization (labelled "Analytical"). A third approach of calculating the APF current references according to [8], has also been used as a benchmark case, and is referred to as BM2. This approach does not explicitly consider the impedance between the busses and calculates the current references to the APF by summing the harmonic load currents, i.e. $i_{\mathrm{AF}}=i_{L 1}^{h}+i_{L 2}^{h}$, which corresponds to $k_{1}$ and $k_{2}$ equal to 1.0 in (15). Furthermore, results from traditional local selective harmonic filtering of load 2 are used as a reference for the THDs in the system, and is labelled as BM1. The harmonics to be mitigated are the $11^{\text {th }}, 13^{\text {th }}, 23^{\text {th }}$ and $25^{\text {th }}$ components. For each case two plots are given; the APF current measured after the LCL filter, and the ideal APF reference from the controllers.

\section{A. Case 1}

Table III lists the configuration of Case 1 and the results from the simulation. The load demand from both propulsion loads are set to $0.22 \mathrm{pu}$. The resulting THDs indicate that the MPC is able to achieve the most effective
Table II: MPC implementation details.

\begin{tabular}{|ll|}
\hline Parameter & Value \\
\hline Time horizon $T$ & $12.5 \mathrm{~ms}$ \\
MPC run cycle & $100 \mathrm{~Hz}$ \\
Discretization $N$ & 220 \\
Integrator & RK4 \\
Hessian & Exact Hessian \\
approximation & \\
Solver & qpOASES \\
Iterations & 5 \\
Stage cost weights & $q_{1}=q_{2}=1000, q_{u}=1, q_{a b c}=0$ \\
APF current limit & $i_{F}^{a p}=1[\mathrm{pu}]$ \\
(APF pu model) & \\
\hline
\end{tabular}

Table III: Configuration and results from Case 1.

\begin{tabular}{|c|c|c|c|c|}
\hline & MPC & Analytical & BM2 & BM1 \\
\hline THD $v_{1}$ & $1.5 \%$ & $1.6 \%$ & $2.0 \%$ & $2.6 \%$ \\
\hline THD $v_{2}$ & $1.7 \%$ & $2.5 \%$ & $2.2 \%$ & $2.3 \%$ \\
\hline $\begin{array}{l}\text { Power load } 1 \\
\text { Power load } 2\end{array}$ & \multicolumn{4}{|c|}{$0.22[\mathrm{pu}]$} \\
\hline
\end{tabular}

Table IV: Configuration and results from Case 2.

\begin{tabular}{|l|crrr|}
\cline { 2 - 5 } \multicolumn{1}{c|}{} & MPC & Analytical & BM2 & BM1 \\
\hline THD v & $3.9 \%$ & $4.7 \%$ & $4.7 \%$ & $6.4 \%$ \\
THD v & $4.1 \%$ & $5.2 \%$ & $4.9 \%$ & $5.6 \%$ \\
\hline Power load 1 & \multicolumn{4}{c|}{$1.0[\mathrm{pu}]$} \\
Power load 2 & \multicolumn{4}{c|}{$1.0[\mathrm{pu}]$} \\
\hline
\end{tabular}

harmonic mitigation at both buses. The analytical controller is better than BM2 for $\mathbf{v}_{1}$, however, results in worse THD for $\mathbf{v}_{2}$. This is a result of the balanced optimization of the generator current harmonics, and the analytical controller is in total slightly better than BM2. All these three control approaches are ensuring a significantly improved THD at bus 1 compared to BM1.

Fig. 6 shows the resulting APF reference and output current for the MPC, the analytical optimization and BM2. As can be seen, the phase and amplitudes for all three controllers are different, and the MPC generates an APF reference current with lower amplitude compared to the two other controllers. The current reference with the analytical optimization has higher amplitude and a different phase compared to BM2, since the shunt and series impedances in the system are accounted for.

Since the MPC is re-initialized before every new cycle, it is able to compensate for model/process mismatch, and this is causing the THD difference between the MPC and the analytical controller. The analytical controller does not have any closed-loop feedback and is not able to compensate for model/process mismatch. BM2 does not have any information about the power system, as the APF reference is constructed directly from the sum of the harmonics to be mitigated from both loads.

\section{B. Case 2}

Table IV lists the configuration and the results from Case 2. The load demand from both propulsion loads are now set to $1.0[\mathrm{pu}$. As can be seen, the MPC clearly results in the lowest THDs at both buses. In this case, the analytical current reference calculation is resulting in poorer harmonic mitigation at bus 2 than BM2 while the conventional BM1 control provides the worst results. 

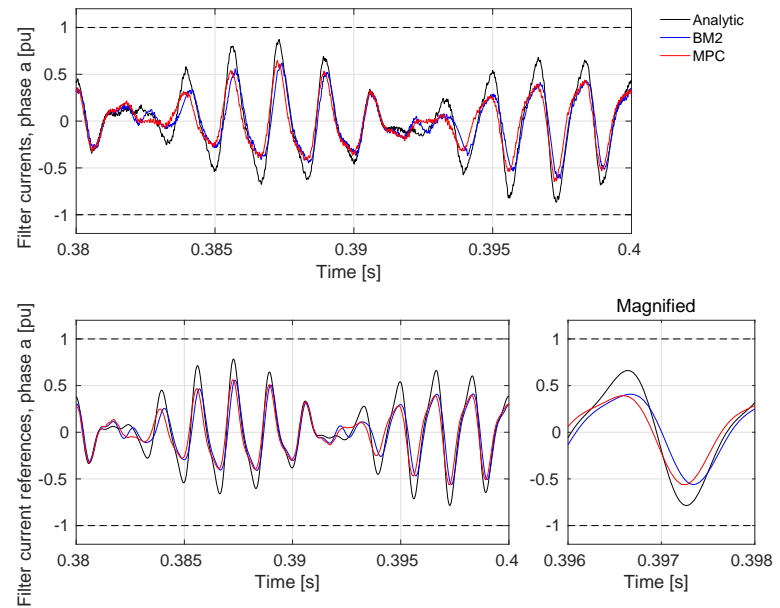

Fig. 6. APF reference and output current (phase a) for Case 1

Fig. 7 shows the resulting APF reference and output current for the MPC, the analytical optimization and BM2. The same as discussed for Case 1 also yields for this case, however now the APF currents are saturated. As the MPC's model includes the APF's current limits as constraints, it is able to optimize the APF performance within the saturation limit. The analytical controller does not have any information regarding the APF's current limits. Hence, the saturation deteriorates the controller's optimality, which in this case results in worse THD for $\mathbf{v}_{2}$ compared to BM2. As the analytical controller employs a higher amplitude and a phase shift compared to BM2, the saturation effects are more severe. It should be noted it is the harmonic current references for the APF that are limited within $1.0 \mathrm{pu}$. Thus, the actual current injected into bus 2 is slightly exceeding this limit due to the fundamental frequency current needed to compensate for losses and balance the dc-bus voltage of the APF.

\section{Conclusion}

This work has analyzed two conceptual methods for optimized system-wide harmonic mitigation of a two-bus power system by an APF. First, it has been shown how an analytical expression for the APF current that will minimize each harmonic component at the two buses can be calculated as a function of the load current harmonics and the system parameters. This serves to demonstrate how the APF can be controlled to benefit the overall system and not only the local point where it is connected. However, the purely analytical approach is sensitive to system parameter variations and does not take into account practical constraints like the current rating of the APF. An MPC-based controller is shown to be able to optimize the APF performance within such constraints. Thus, the MPC demonstrated clear advantages over the analytically obtained current references, related to robustness and adaptive behavior, resulting in lower THDs at both bus voltages in the presented simulations.

\section{Acknowledgement}

This work has been carried out at the Centre for Autonomous Marine Operations and Systems (AMOS), sponsored by the Norwegian Research Council. The work was supported by Ulstein Power \& Control AS and the Research Council of Norway, Project number 241205.
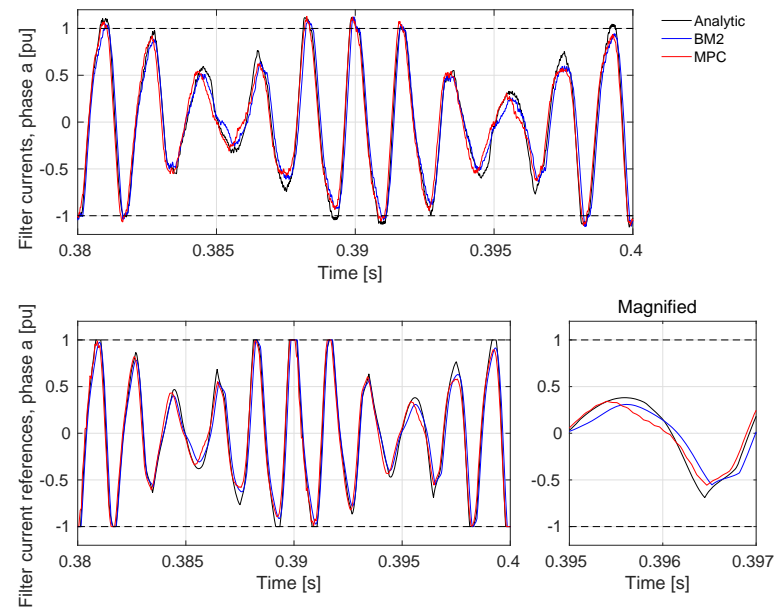

Fig. 7. APF reference and output current (phase a) for Case 2

\section{References}

[1] G. K. Singh, "Power system harmonics research: a survey," in European Transactions on Electrical Power, vol. 19, no. 2, March 2009, pp. 151-172

[2] P. Mattavelli, "A Closed-Loop Selective Harmonic Compensation for Active Filters," in IEEE Trans. on Ind. Appl., vol. 37, no. 1, January/February 2001, pp. 81-89

[3] H. Akagi, E. Watanabe, and M. Aredes, "Instantaneous Power Theory and Applications to Power Conditioning," Wiley/IEEE Press Series on Power Engineering, 2007

[4] W. M. Grady, M. J. Samotyj, A. H. Noyola, "Minimizing Network Harmonic Voltage Distortion with an Active Power Line Conditioner," in IEEE Transactions on Power Delivery, vol. 6, no. 4, October 1991, pp. 1690-1697

[5] E. Skjong, M. Molinas, T. A. Johansen, "Optimized Current Reference Generation for System-level Harmonic Mitigation in a Diesel-Electric Ship," in Proc. of the 2015 IEEE Int. Conf. on Industrial Technology, ICIT 2015, Seville, Spain, 17-19 March 2015, pp. 2314-2321

[6] E. Skjong, M. Molinas, T. A. Johansen, R. Volden, "Shaping the Current Waveform of an Active Filter for Optimized System Level Harmonic Conditioning," in Proc. of the $1^{\text {st }}$ Int. Conf. on Vehicle Technology and Intelligent Transportation Systems, VEHITS 2015, Lisbon, Portugal, 20-22 May 2015, pp. 98-106

[7] E Skjong, M. Ochoa-Gimenez, M. Molinas, T. A. Johansen, "Management of harmonic propagation in a marine vessel by use of optimization," in Proc. of the 2015 IEEE Transportation Electrification Conf. and Expo, ITEC 2015, Dearborn, Michigan, USA, 14-17 June 2015, 8 pp.

[8] A. R. Årdal, E. Skjong, M. Molinas, "Handling System Harmonic Propagation in a Diesel-Electric Ship with an Active Filter," in Proc. of the 2015 Int. Conf. on El. Syst. for Aircraft, Railway, Ship Propulsion and Road Vehicles, ESARS 2015, Aachen, Germany, 3-5 March 2015, 6 pp.

[9] M. R. Patel, "Shipboard Electrical Power Systems," Taylor \& Francis, 2011

[10] J. Rawlings, D. Mayne, "Model Predictive Control: Theory and Design," Nob Hill Pub. 2009

[11] J. A. Suul, "Control of Grid Integrated Voltage Source Converter under Unbalanced Conditions," PhD Thesis, Norwegian University of Science and Technology, 2012

[12] B. Houska, H. J. Ferreau, M. Diehl, "ACADO Toolkit An Open Source Framework for Automatic Control and Dynamic Optimization," in Optimal Control Applications and Methods, vol. 32, no. 3, May/June 2011, pp. 298-312

[13] B. Houska, H. J. Ferreau, M. Diehl, "An Auto-Generated Real-Time Iteration Algorithm for Nonlinear MPC in the Microsecond Range," in Automatica, vol. 47, no. 10. October 2011, pp. 2279-2285 